\title{
Role of orexin-2 and CB1 receptors within the periaqueductal gray matter in lateral hypothalamic-induced antinociception in rats.
}

$\underline{\text { Esmaeili MH}}{ }^{1}, \underline{\text { Reisi Z }}, \underline{\text { Ezzatpanah S }}, \underline{\text { Haghparast A. }}$.

Department of Physiology, Cellular and Molecular Research Center, Qazvin University of Medical Sciences, Qazvin bSchool of Medicine, Neuroscience Research Center, Shahid Beheshti University of Medical Sciences, Tehran, Iran.

\begin{abstract}
Orexin plays an important role in pain modulation. Orexin-1 and orexin-2 receptors (Ox1r and Ox2r) are found at high density in the ventrolateral periaqueductal gray matter (vIPAG). Our previous study showed that chemical stimulation of the lateral hypothalamus with carbachol induces antinociception in the tail-flick test, a model of acute pain, and Ox1r-mediated antinociception in the vIPAG is modulated by the activity of vlPAG CB1 receptors. In the current study, TCS OX2 29, an Ox2r antagonist $(5,15,50,150$, and $500 \mathrm{nmol} / \mathrm{l})$, was microinjected into the vlPAG 5 min before the administration of carbachol (125 nmol/l). TCS OX2 29 dose dependently reduced carbachol-induced antinociception. In a second set of experiments, animals were treated with carbachol 5 min after intra-vlPAG administration of $15 \mathrm{nmol} / 1$ TCS OX2 29 and $1 \mathrm{nmol} / 1$ AM251 (a selective CB1 receptor antagonist), or $150 \mathrm{nmol} / 1$ TCS OX2 29 and $10 \mathrm{nmol} / 1$ AM251. The findings showed that the antinociceptive effect of orexin is partially mediated by activation of vIPAG Ox 2 receptors. Furthermore, the administration of ineffective doses of $\mathrm{Ox} 2$ and $\mathrm{CB} 1$ receptor antagonists reduced the lateral hypothalamus-induced antinociception. It seems that $\mathrm{Ox} 2$ and $\mathrm{CB} 1$ receptors act through different pathways and Ox2r-mediated antinociception is not dependent on CB1 receptor activity.
\end{abstract}

